\title{
Football match spectator sound exposure and effect on hearing: A pretest-post-test study
}

\author{
De Wet Swanepoel, James W Hall III
}

Objectives. To determine (i) noise exposure levels of spectators at a FIFA 2010 designated training stadium during a premier soccer league match; and (ii) changes in auditory functioning after the match.

Methods. This was a one-group pretest-post-test design of football spectators attending a premier soccer league match at a designated FIFA 2010 training stadium in Gauteng, South Africa.

Individual spectator noise exposure for the duration of the football match and post-match changes in hearing thresholds were measured with pure-tone audiometry, and cochlear functioning was measured with distortion product otoacoustic emissions (DPOAEs).
Results. The average sound exposure level during the match was 100.5 LAeq (dBA), with peak intensities averaging 140.4 $\mathrm{dB}(\mathrm{C})$. A significant $(p=0.005)$ deterioration of post-match hearing thresholds was evident at $2000 \mathrm{~Hz}$, and post-match DPOAE amplitudes were significantly reduced at 1266,3163 and $5063 \mathrm{~Hz}$ ( $p=0.011,0.019,0.013$, respectively).

Conclusions. Exposure levels exceeded limits of permissible average and peak sound levels. Significant changes in postmatch hearing thresholds and cochlear responsiveness highlight the possible risk for noise-induced hearing loss. Public awareness and personal hearing protection should be prioritised as preventive measures.

S Afr Med J 2010; 100: 239-242..
Football fever is rising in anticipation of the quadrennial 2010 FIFA World Cup event. Half a million international supporters are expected to converge on South Africa for the historic event, to be hosted in Africa for the first time. Spectators will be greeted by a distinctively African sporting spectacle uniquely characterised by sounds produced by sports fans blowing the horn-like instrument known as the vuvuzela. ${ }^{1}$ Cherished by local supporters, the vuvuzela produces a characteristically loud, reverberant sound that can be heard for miles around the stadium. In the recent 2009 Confederations Cup held in South Africa, the international football community noticed this phenomenon. ${ }^{1}$ Despite complaints from international teams, spectators and commentators about the uninterrupted loudness of the vuvuzela, FIFA approved it as part of the signature 2010 World Cup to be held in Africa. ${ }^{1,2}$

The actual sound output created by the vuvuzela was recently reported to reach dangerously high levels, averaging $131 \mathrm{~dB}(\mathrm{~A})$ at the horn opening and $113 \mathrm{~dB}(\mathrm{~A})$ at a 2-metre distance from the vuvuzela. ${ }^{2}$ These values exceed international directives on exposure limits in occupational settings. Although there is significant variability in individual susceptibility, prolonged or regular exposure poses a significant risk for noise-induced hearing loss. ${ }^{3-5}$ Recreational activities with excessively loud sound levels are an increasingly important public health care concern for developing permanent

Department of Communication Pathology, University of Pretoria, and Callier Center for Communication Disorders, University of Texas at Dallas, USA De Wet Swanepoel, PhD

Department of Communicative Disorders, University of Florida at Gainesville, Fla, USA, and Department of Communication Pathology, University of Pretoria James W Hall III, PhD

Corresponding author: D Swanepoel (dewet.swanepoel@up.ac.za) sensorineural hearing loss..$^{5-8}$ We aimed to determine (i) noise exposure levels of spectators at a FIFA 2010 designated training stadium during a premier soccer league match; and (ii) changes in auditory functioning soon after the match.

\section{Methods}

Ethical clearance was received from the University of Pretoria (September 2009), and we obtained permission from the City of Tshwane to conduct the study at the specific stadium. Each participant signed an informed consent form.

\section{Study population}

Spectators at a South African premier soccer league match held in October 2009 at a FIFA 2010 designated training stadium with a 30000 -seat capacity were sampled to participate in the study. Eleven participants ( 2 female) with an average age of 27.2 years (range 20 - 40 years) were drafted for the study. Participants were provided with a free ticket to the match. The left and right ear of each participant was assessed before and after the match. Only one of the 22 ears assessed did not have normal hearing averaged across all frequencies $(<20 \mathrm{~dB} H \mathrm{HL})$ because of a perforated tympanic membrane. As a result, preand post-match hearing data were collected from 21 ears (11 participants). Participants were not allowed to consume alcohol before the post-match assessment was completed.

\section{Data collection}

All participants were assessed by four audiology clinicians within 3 hours before attending the football match. The assessment included an otoscopic examination and tympanometry to rule out external and middle ear abnormalities. One left ear ( $1 / 22$ ears) was excluded because of a tympanic membrane perforation. All remaining ears of the 11 subjects were tested with pure-tone audiometry, highfrequency pure-tone audiometry, and distortion product oto-acoustic emissions (DPOAEs). Pure-tone audiometry was 
conducted with a calibrated diagnostic type 1 audiometer in a sound-proof booth. Thresholds were determined using a bracketing method ( $10 \mathrm{~dB}$ down and $5 \mathrm{~dB}$ up), commencing at $30 \mathrm{~dB}$ HL with threshold defined as the lowest level where the patient could correctly respond to $50 \%$ of presentations at that intensity. The conventional audiometric frequency range, including 250, 500, 1 000, 2 000, 3 000, 4 000, 6000 and 8000 $\mathrm{Hz}$, was assessed using TDH-49 supra-aural earphones, while the high-frequency range, including 10000,12 500, 14000 and $16000 \mathrm{~Hz}$, was assessed using Sennheiser HDA200 circumaural earphones. DPOAEs, which indicate outer hair cell integrity of the cochlea, were also measured (in 21 ears) using a BioLogic Scout system across $\mathrm{f} 2$ frequencies including 1 008, 1 266, 1 570, 1992, 2 508, 3 164, 4 008, 5 063, 6352 and $8016 \mathrm{~Hz}$. A fixed f2:f1 ratio of 1.22 was maintained with the primary tones presented at 65 (f1) and 55 (f2) dB SPL. DPOAE measurements of $2 \mathrm{f} 1$ - f2 amplitude were plotted as a function of the $\mathrm{f} 2$ frequency. Each DPOAE measurement was replicated without removing the probe from the ear canal.

All auditory tests were repeated in exactly the same manner as the pre-match testing between 1 and 3 hours after the football match ended. The order of conventional and highfrequency pure-tone audiometry and DPOAE tests were counter-balanced and the same clinician tested each participant in the pre- and post-match assessments.

During the match, 10 of the 11 participants wore personal sound exposure meters (doseBadges), fixed to their shoulder, which continuously measured each individual's noise exposure. The personal sound exposure meters were set to record sound levels before entering the stadium and were switched off after participants had exited the stadium. The participants were assigned seats in pairs of two (except for one group of three, the third of whom did not wear a doseBadge) distributed across the stadium to include exposure levels close to the field, in the middle of the stadium and right at the back of the stadium. Two of the five pairs of participants blew vuvuzelas during the match.

\section{Data analyses}

The exposure levels measured by each of the 10 personal sound level devices were downloaded and analysed according to the time of exposure for each participant. Hearing thresholds measured in pre- and post-test conditions were compared along with averaged DPOAE amplitudes of the two pre-match recordings and the two post-match recordings, using the Wilcoxon signed-rank test for repeated measurements on a single sample. The $\mathrm{f} 2$ frequency in the DPOAE measurements denotes the cochlear frequency region evaluated. DPOAE amplitudes are therefore documented with respect to the f2 frequency.

\section{Results}

Table I summarises the personal sound exposure levels for participants for the duration of their exposure as spectators. The average sound level experienced by participants during the almost 2-hour exposure was 100.5 LAeq (dBA). Converting this average sound exposure value to a daily personal exposure level related to an 8-hour work day translates to an average of 94.4 dB (LEP,d). The peak sound level exceeded $140 \mathrm{~dB}$ for 8 of the 10 participants, with a maximum peak level reaching 144.2 $\mathrm{dB}(\mathrm{C})$. The 4 subjects who blew vuvuzelas had the highest LAeq exposure levels, all in excess of $100 \mathrm{~dB}(\mathrm{~A})$, and their exposure to peak sound levels was in excess of $140 \mathrm{~dB}(\mathrm{C})$.

The changes in pure-tone audiometry thresholds preand post-match are presented in Fig. 1. More than 50\% of hearing thresholds at 250, 2 000, 3 000, 4000 and $6000 \mathrm{~Hz}$ demonstrated a post-match deterioration. The only statistically significant post-match deterioration, however, was at $2000 \mathrm{~Hz}$ $(p=0.005)$, with an average threshold elevation (shift) of $3.4 \mathrm{~dB}$ $( \pm 4.2$ standard deviation (SD)).

DPOAE amplitudes were recorded with acceptably low noise floor levels across pre- and post-match measurements. Figs 2 and 3 illustrate the average change in DPOAE amplitudes pre- and post-match. There is a notable post-match decrease in DPOAE amplitudes, which was statistically significant at

\begin{tabular}{|c|c|c|c|c|}
\hline Participants & Time of exposure (min) & LAeq dB(A) & LEP,d & LPeak dB(C) \\
\hline $1^{*}$ & 115 & 100.3 & 94.1 & 140.8 \\
\hline 2 & 114 & 98 & 91.8 & 141.5 \\
\hline 3 & 115 & 95.8 & 89.6 & 140.6 \\
\hline 4 & 114 & 98.5 & 92.3 & 142.1 \\
\hline $5^{*}$ & 114 & 103.9 & 97.7 & 142.3 \\
\hline 6 & 115 & 99.2 & 93.1 & 142.3 \\
\hline $7^{*}$ & 125 & 103.5 & 97.6 & 144.2 \\
\hline $8^{*}$ & 114 & 106.9 & 100.7 & 142.2 \\
\hline 9 & 124 & 99.2 & 93.4 & 135.3 \\
\hline 10 & 124 & 99.5 & 93.6 & 132.7 \\
\hline Average & 117.4 & 100.5 & 94.4 & 140.4 \\
\hline $\mathrm{SD}$ & 4.8 & 3.3 & 3.3 & 3.6 \\
\hline Min. & 114.0 & 95.8 & 89.6 & 132.7 \\
\hline Max. & 125.0 & 106.9 & 100.7 & 144.2 \\
\hline \multicolumn{5}{|c|}{$\begin{array}{l}\text { *Participants blowing a vuvuzela. } \\
\text { LAeq = the equivalent noise exposure averaged over the duration of exposure, in other words the average sound intensity for the duration of exposure if it was the same volume } \\
\text { throughout; } \mathrm{LEP}, \mathrm{d}=\text { the worker's daily noise exposure, in other words the specific amount of noise exposure for the duration of exposure averaged over an 8-hour working day; } \mathrm{LPeak} \\
\mathrm{dB}(\mathrm{C})=\text { the peak level of the sound pressure measured over the duration of exposure; } \mathrm{SD}=\text { standard deviation. } \\
\text { European Parliament directive on noise levels: }{ }^{3} \text { upper action level when } \mathrm{LEP}, \mathrm{d}=85 \mathrm{~dB}(\mathrm{~A}) \text { and } / \text { or } \mathrm{LPeak}=137 \mathrm{~dB}(\mathrm{C}) \text {; exposure limit when } \mathrm{LEP}, \mathrm{d}=87 \mathrm{~dB}(\mathrm{~A}) \text { and } / \text { or } \mathrm{LPeak}=140 \mathrm{~dB}(\mathrm{C}) \text {. }\end{array}$} \\
\hline
\end{tabular}




\section{Original Articles}

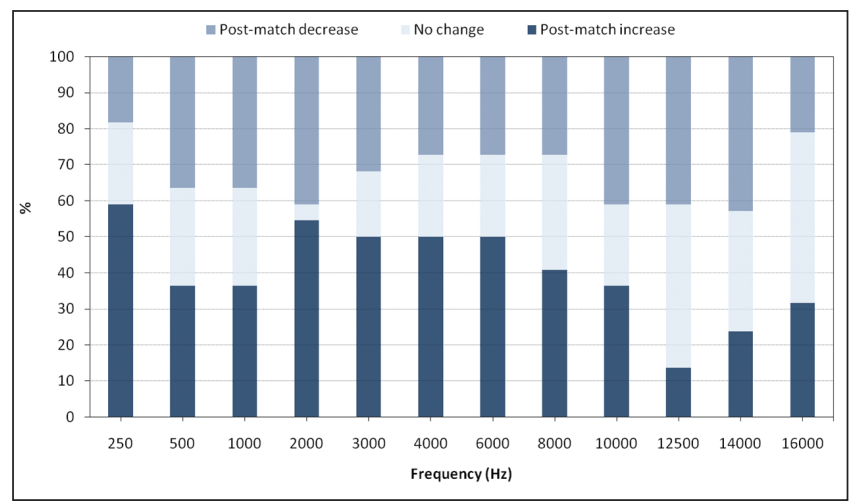

Fig. 1. Distribution of pure-tone audiometry threshold changes pre-and post-match (threshold increase $=$ deterioration in hearing).

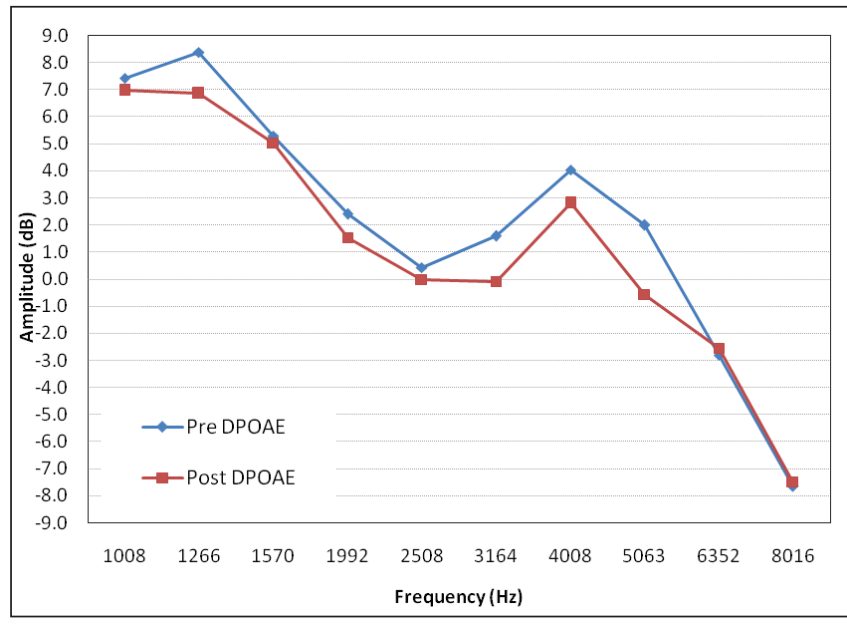

Fig. 2. Average DPOAE amplitudes pre-and post-match (21 ears).

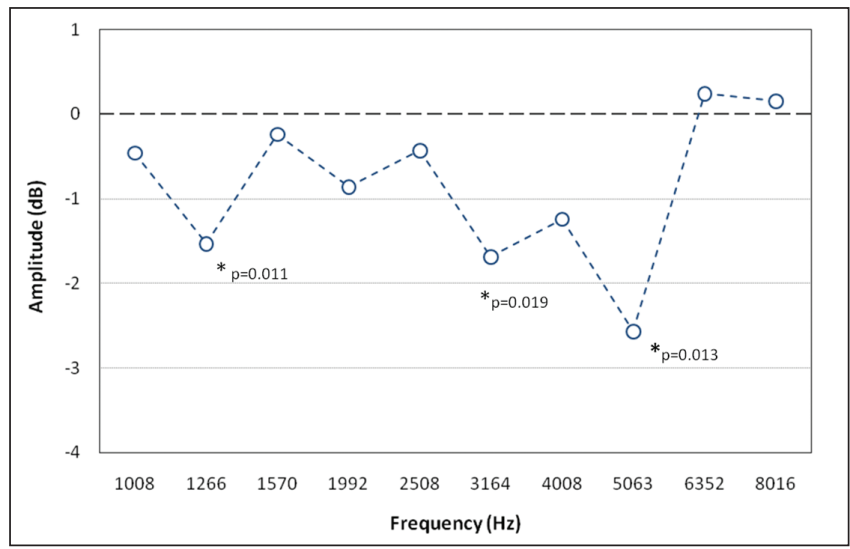

Fig. 3. Average pre- and post-match DPOAE amplitude difference (21 ears) ( ${ }^{*}$ significant post-match differences).

1266,3163 and $5063 \mathrm{~Hz}$, with an average decrease of 1.5, 1.7 and $2.6 \mathrm{~dB}$, respectively. Fig. 4 indicates the distribution of DPOAE amplitudes post-match presenting with a decrease in amplitude. Overall, $63 \%$ of DPOAE amplitudes across $\mathrm{f} 2$ frequencies decreased post-match, and $73 \%$ of amplitudes at 1266,3164 and $5063 \mathrm{~Hz}$ demonstrated a decrease post-match averaging $2.6,3.1$ and $4.2 \mathrm{~dB}$, respectively.

\section{Discussion}

The exposure to high-intensity sound for all study participants during the football match was at high average levels (100.5 LAeq), with peak energy exposures exceeding $140 \mathrm{~dB}(\mathrm{C})$ in the

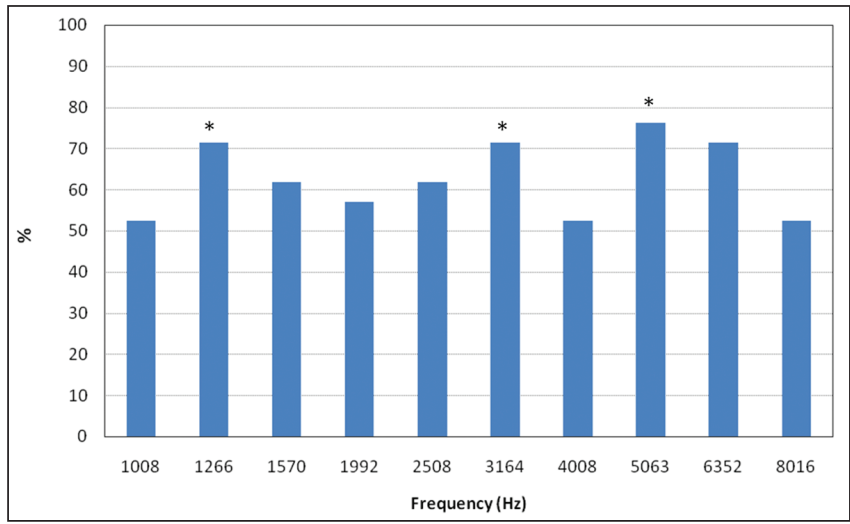

Fig. 4. Distribution of reduced post-match DPOAE amplitudes (21 ears) ( ${ }^{*}$ significant post-match differences).

majority $(8 / 10)$ of participants. The 4 participants in this study who blew vuvuzelas had the highest levels of average noise exposure. Post-match auditory tests indicated a significant average elevation in hearing thresholds at $2000 \mathrm{~Hz}$, and DPOAE amplitudes were significantly reduced at 1266,3163 and $5064 \mathrm{~Hz}$. Although auditory changes probably reflect a temporary shift in hearing threshold and DPOAE amplitude,, 7 they demonstrate that auditory physiology is affected by the sound exposure levels experienced at a football match where vuvuzelas are a major source of acoustic energy.

To contextualise the sound exposure levels experienced by the participants and the associated risks involved, they should be considered within occupational noise exposure legislation. According to the Physical Agents (Noise) Directive 2003/10/ $\mathrm{EC}^{3}$ issued by the European Parliament in 2003, there are two criteria to consider in terms of sound exposure and risk of hearing loss. The first is the average exposure level over the specific duration of exposure normalised to an 8-hour working day (LEP,d), and the second is the peak energy recorded during the duration of exposure (LPeak). These values were compared against regulated criteria (Table I) to determine the associated risk and necessary actions to be taken. The exposure levels in this study exceeded the specified exposure limit of $87 \mathrm{~dB}(\mathrm{~A})$ for all participants by between 3 and $14 \mathrm{~dB}$. The LPeak levels also exceeded the regulated exposure limit of $140 \mathrm{~dB}(\mathrm{C})$ in the majority $(8 / 10)$ of participants. The Exposure Limit Action Level specified by Directive 2003/10/EC specifies that under no circumstances shall the exposure of the worker (taking into account hearing protection worn) exceed these limits (Physical Agents (Noise) Directive, 2003). South African standards for occupational noise stipulate personal hearing protection for workers exposed to levels of $85 \mathrm{dBA}$ and above. ${ }^{9}$ Considering the logarithmic nature of the decibel scale, implying a doubling of hearing loss risk with every $3 \mathrm{~dB}$ increase, ${ }^{5}$ participants in this sample were exposed to high-intensity sound far exceeding the current legislated average exposure and peak exposure levels for occupational noise.

The significant reduction in DPOAE amplitudes after the match, compared with a significant deterioration in hearing thresholds only at $2000 \mathrm{~Hz}$, is in agreement with previous studies indicating that OAE measurements can identify early cochlear damage not yet reflected in hearing thresholds., ${ }^{70-12}$ Although not documented in this study, these changes are probably temporary, as demonstrated by Bhagat and 
Davis, ${ }^{7}$ but typically precede permanent changes in cochlear functioning and pure-tone hearing thresholds. ${ }^{12}$ Since OAEs directly measure the responsiveness of the outer hair cells of the cochlea, which is the primary site of the lesion in noiseinduced hearing loss, these measures can identify changes in auditory functioning before conventional measures such as pure-tone audiometry.,10,12

The vuvuzela is an iconic symbol of football culture in South Africa and is bound to enthral spectators from around the world at the 2010 FIFA World Cup. ${ }^{2}$ We cannot specify the contribution of the vuvuzela to the overall sound level recorded during the football match, although a preliminary report on its sound output levels ${ }^{2}$ and the exposure levels recorded for the four participants blowing vuvuzelas in this study demonstrate its high-intensity sound. In addition to this, concerns raised independently by representatives of international football teams, football match spectators and sports commentators about the almost constant loud sound produced during the match by thousands of vuvuzelas implicate the instrument as a major factor in the recorded noise levels. Most importantly, the findings of this study demonstrate a real risk of noise-induced hearing loss. Official match stadiums for the 2010 FIFA World Cup will house 90000 spectators, three times more than the stadium used in this study. It is reasonable to suspect that sound intensity will be even higher in the larger official venues. The findings of this study support the recommendation for hearing protection of football match spectators. At the least, preventive measures, such as public awareness and personal hearing protection, are warranted.
The authors would like to acknowledge Mr Shadrack Mngemane, Ms Silindile Molemong, Ms Hilda Mkwanazi and Mr Sizwe Tutshini for their valuable assistance in the data collection for this study.

\section{References}

1. Fédération Internationale de Football Association (FIFA). Vuvuzela: a symbol of South Africa. 19 June 2009. http://www.fifa.com/confederationscup/news/newsid=1073689.html (accessed 28 July 2009).

2. Swanepoel D, Hall III JW, Koekemoer D. Vuvuzela - good for your team, bad for your ears. S Afr Med J 2010; 100: 99-100.

3. Directive 2003/10/EC of the European Parliament and of the Council of 6 February 2003 on Directive 2003/10/EC of the European Parliament and of the Council of 6 February 2003 on
the minimum health and safety requirements regarding the exposure of workers to the risks arising from physical agents (noise). Official Journal of the European Union L42 of 15.2.2003, p. 38 .

4. ISO (International Organization for Standardization). Acoustics - Determination of Occupational Noise Exposure and Estimation of Noise-Induced Hearing Impairment. 2nd ed. Geneva: ISO, 1990 (Reference No. ISO 1999 1990(E))

5. Clark WW, Bohne BA. Effects of noise on hearing. JAMA 1999; 281: 1658-1659.

6. Potier M, Hoquet C, Lloyd R, Nicolas-Puel C, Uziel A, Puel JC. The risks of amplified music for disc-jockeys working in nightclubs. Ear Hear 2009; 30: 291-293.

Bhagat SP, Davis AM. Modification of otoacoustic emissions following ear-level exposure to MP3 player music. Int I Audiol 2008; 47: 751-760.

8. Vinck BM, Van Cauwenberge PB, Leroy L, Corthals P. Sensitivity of transient evoked and distortion product otoacoustic emissions to the direct effects of noise on the human cochlea. Audiology 1999; 38: 44-52

9. The Measurement and Assessment of Occupational Noise for Hearing Conservation purposes. Pretoria: South African National Standards, 2004 (SANS10083:2004).

10. Attias J, Horovitz G, El-Hatib N, Nageris B. Detection and clinical diagnosis of noise-induced hearing loss by otoacoustic emissions. Noise Health 2001; 3: 19-31.

11. Korres GS, Balatsouras DG, Tzagaroulakis A, Kandiloros D, Ferekidou E, Korres S. Distortion product otoacoustic emissions in an industrial setting. Noise Health 2009; 11: 103-110.

12. Ceranic B. The value of otoacoustic emissions in investigations of noise damage. Audiol Med 2007; 5: 10-24.

Accepted 2 March 2010. 